\title{
Morphology of the Head and Neck of the Macaque Monkey: The Muscles of Mastication and the Mandibular Division of the Trigeminal Nerve
}

\author{
DAVID J. SCHWARTZ and DONALD F. HUELKE \\ Department of Anatomy, University of Michigan, Ann Arbor, Michigan
}

Today young Rhesus monkeys, especially Macaca mulatta, are widely used for dental and medical research, and there is no doubt that they will be used even more in the future. Certain regions or systems of this animal-brain, spinal cord, extremity musculature-have been adequately described, whereas only a meager amount of information has been written about the detailed anatomy of the head, neck, torso, and most of the neurovascular systems of the extremities. In the head and neck, experimental procedures have been carried out, with little being said about the "normal" anatomy of the animal. However, the most concise work on the Rhesus monkey, by Hartman and Strauss, ${ }^{1}$ has been used as the standard reference text by many. Most reports on the anatomy of laboratory animals lack morphologic detail, are superficial in their coverage, sketchy in their descriptions, or even incorrect. Many of the descriptions appear to have been based on only a few specimens. Since anatomical details on the Macaque are noticeably lacking, it is our intent to review its morphology in detail, beginning with the craniofacial area.

\section{Materials and Methods}

Since most monkeys used for experimental work, in almost all laboratories today, are immature, it was felt that they, and not the adult specimens, should be used in our morphologic studies. For the muscle dissection, we used approximately 20 heads of monkeys ranging in estimated age from 6 months to 2 years (primary to early mixed dentition). Some of these specimens plus an additional 15 heads were used for the nerve dissections. Gross dissection was supplemented by the occasional use of the binocular microscope for details, and microscopic sections were made of certain tissues to verify their structure.

\section{Results}

Masticatory musculature: Masseter.-The masseter muscle was deep under the skin and the upper fibers of the platysma muscle; it was partially covered by the parotid gland and the buccal pouch. The muscle was divided into a superficial and

This paper was presented in part at the fortieth meeting of the International Association for Dental Research, March 15-18, 1962, St. Louis, Missouri.

This investigation was supported in part by USPHS research grants D-895 and D-1123, and by part-time dental student research fellowships from the National Institute of Dental Research, National Institutes of Health, Bethesda, Maryland.

Received for publication January 2, 1963. 
a deep head on the basis of the direction of the muscle fibers and their distinct arrangement into two groups (Fig. 1). The superficial portion was the larger; it arose by tendinous fibers from the inferior and medial surface of the anterior half of the zygomatic arch, extending from an area forward of the zygomaticomaxillary suture to near the zygomaticotemporal suture. The fibers of the superficial head passed downward and backward to insert into the entire lower half of the lateral surface of the ramus and angle of the mandible. It was noted that the insertion was much stronger at its margins than over the rest of the area of insertion. The deep head of

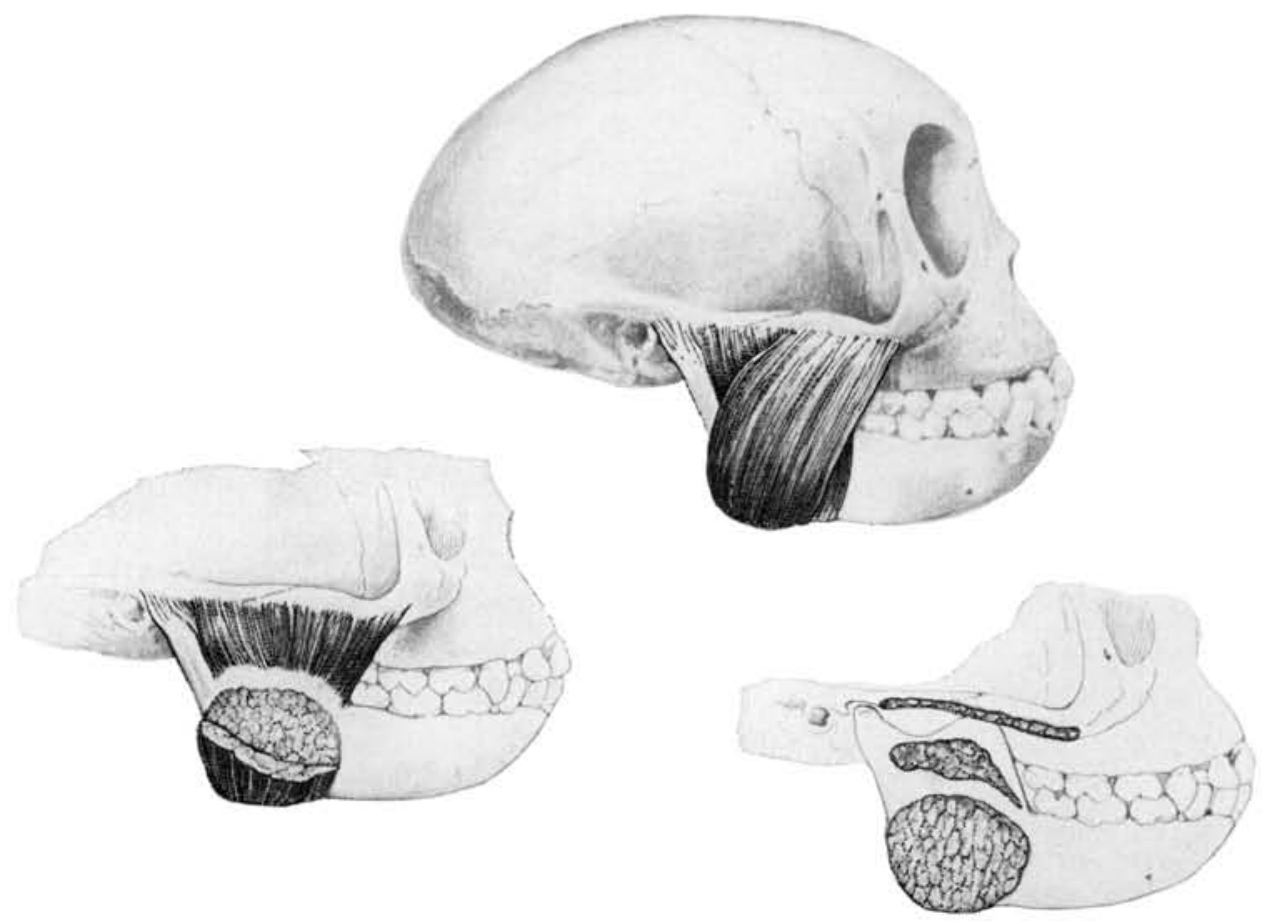

Fig. 1.-The masseter muscle of the Macaque. Note the posterior fibers of the deep head arising from the capsule of the temporomandibular joint.

the masseter took origin from the posterior three-fourths of the zygomatic arch and the lateral and anterior aspects of the temperomandibular joint capsule. These fibers passed almost directly downward, crossing beneath the superficial head at about a $60^{\circ}$ angle, to insert into the upper half of the ramus of the mandible behind and below the coronoid process. This area of insertion was indicated on the bone by a subincisural fossa, located directly beneath the mandibular notch and coronoid process, and it is here that the bulk of the deep head attached. These fibers were loosely attached to the fossa area; however, on the inferior border of the fossa the attachment was noted to be quite strong. Anteriorly, where the superficial head overlay the deep head, there was no clear-cut separation of the two, for here the fibers of both heads intermingled. The most medial of these fibers blended with the temporalis muscle along the anterior border of the ramus and at the base of the coronoid process. 
Temporalis muscle.-The temporalis muscle lay beneath the skin and the deep fascia on the side of the cranium. It was quite thick anteriorly and thinner posteriorly, filling the temporal fossa. The muscle was incompletely divided into two heads, a thicker deep portion and a thinner superficial layer. The temporalis arose from an area outlined by the temporal line above, the temporal crest anteriorly, the infratemporal crest and adjacent sphenoid bone below, and from the overlying deep fascia (Fig. 2).

From this extensive origin, the fibers of the temporalis muscle passed deep into the zygomatic arch to converge on the coronoid process and ramus of the mandible. The posterior fibers traveled forward and curved over the root of the zygomatic arch to a fleshy insertion. The fibers from the deep head inserted on the medial margin of the mandibular notch and medial surface of the coronoid process, while those fibers

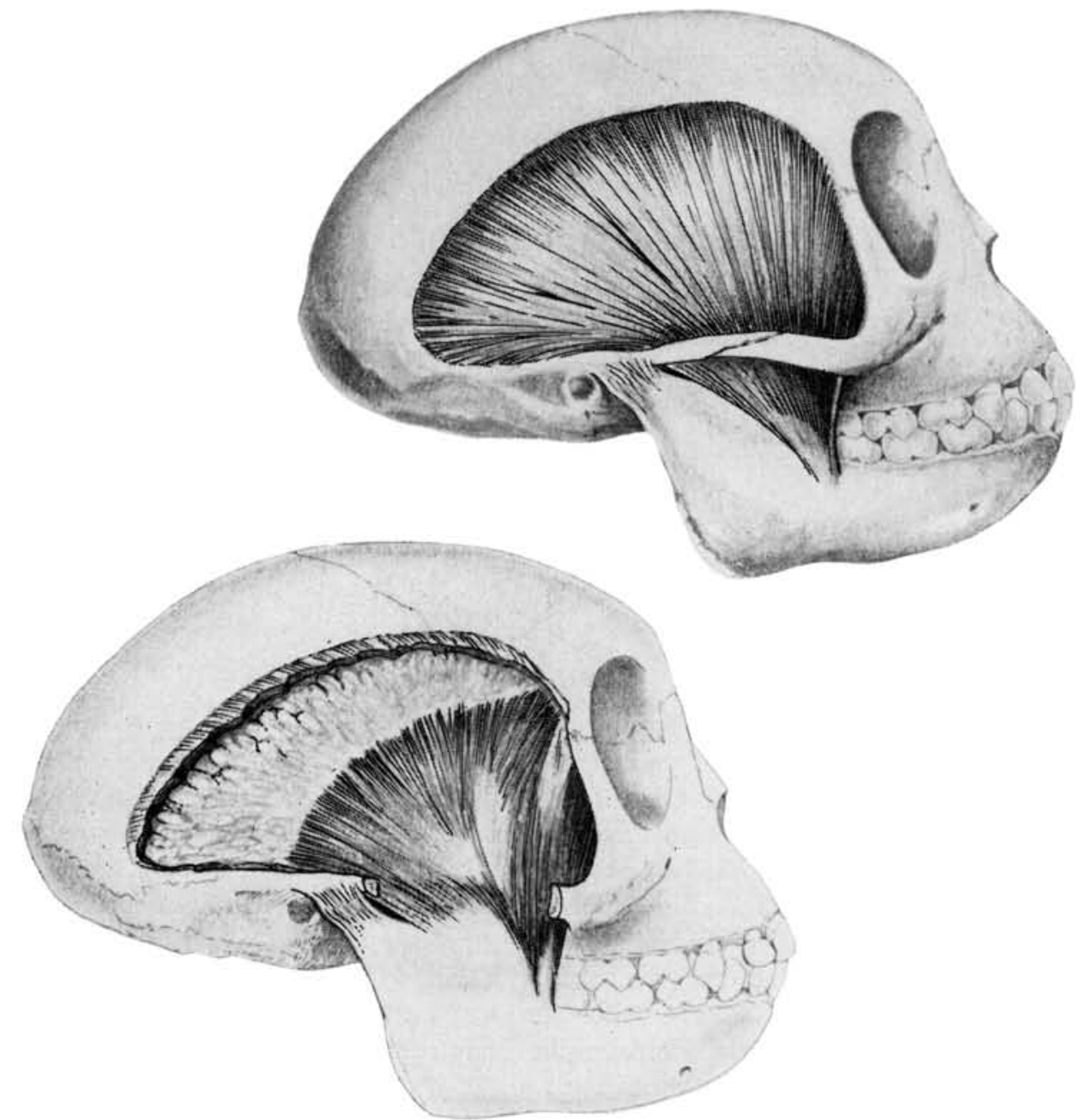

FIG. 2.-The temporalis muscle. Note the extensive attachment along the mandibular notch and along the anterior ramus of the mandible. 
of the superficial head inserted on the lateral margin of mandibular notch and lateral surface of the coronoid process. Almost all of the mandibular notch had temporalis muscle fibers attached to it. The middle fibers of both heads had a tendinous and fleshy insertion on the anterior border of coronoid process and the upper part of the ramus. The fibers of the deep head inserted on the medial surface of the ramus, while fibers of the superficial head inserted on the lateral surface in the same relative area. The anterior fibers of both heads inserted by a strong heavy tendon along the
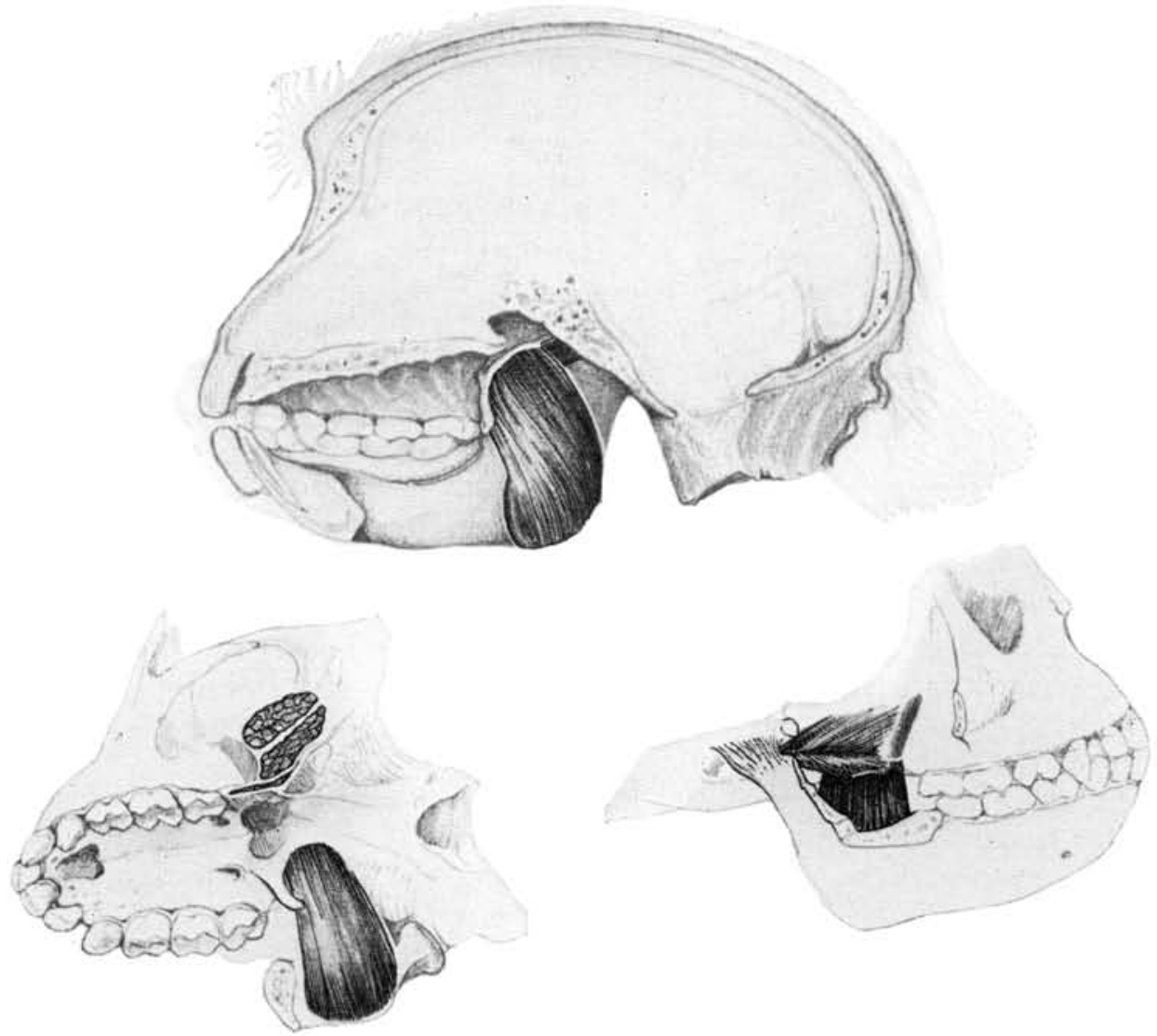

FIG. 3.-The attachments of the medial and lateral pterygoid muscles

anterior border of the ramus and to a tubercle on the posterior part of the oblique line. This tendon passed near the pterygomandibular raphe and was bound to it by a fascial band.

Medial pterygoid.-The medial pterygoid was a thick quadrilateral muscle situated between the medial surface of the ramus and the mandible and the lateral pharyngeal wall. Its fibers ran almost parallel to those of the superficial head of the masseter. The medial pterygoid muscle originated from the pterygoid fossa, between the medial and lateral pterygoid plates (Fig. 3). Its bulk filled the entire depth of the pterygoid fossa. Beyond the fossa other fibers arose from the inferior portion of the lateral 
surface of the lateral pterygoid plate and the immediately adjacent area of the palatine and maxillary bones. Fibers passed downward and slightly backward to their insertion on the medial surface of the angle of the mandible beneath the mandibular foramen and onto the base of the mandible at the angle. This was a heavy muscle throughout, being quite thick even at its lowest attachment (Fig. 5).

Lateral pterygoid muscle.-The lateral pterygoid was the smallest of the four muscles of mastication; it lay deep to the ramus of the mandible and the temporalis tendon. The upper part of the muscle arose from the infra-temporal crest and infratemporal fossa of the sphenoid bone (Fig. 3). The lower portion of the muscle arose from the lateral surface of the lateral pterygoid plate. There was considerable blending and interdigitating of both heads near their insertion. The lower head inserted primarily on the pterygoid fossa of the mandible and on the anteromedial surface of the neck of the condyle, with some fibers going to the capsule of the temporamandibular joint. The upper head inserted primarily on the capsule of the temperomandibular joint, with some fibers attaching to the pterygoid fossa and to the articular disk of the joint. Those fibers inserting into the disk are given the special name of "sphenomeniscus muscle."

The accessory muscles of mastication.-The digastric muscle was composed of one anterior and two posterior bellies; these three bellies were interconnected to each other by intermediate tendons (Fig. 4). The posterior belly lay deep to the parotid gland and to the sternomastoid muscle and was immediately superficial to the carotid sheath. The anterior belly lay deep to the platysma muscle and was partially covered posteriorly by the submandibular gland. The posterior belly of the digastric muscle arose from the mastoid area of the skull, $4-5 \mathrm{~mm}$. posterior and medial to the external acoustic meatus. No fossa or tubercle marked this origin of the posterior digastric belly. From its origin the muscle passed forward and downward as a thin, laterally compressed band; it crossed the carotid sheath obliquely and became tendinous. The intermediate tendon developed within the posterior belly of the digastric. Medial to the mandibular angle the tendon lay against the medial pterygoid muscle. The tendon passed forward to join its fellow of the opposite side to form a U-shaped tendinous arch-a "digastric sling." This arch was strong and heavy and had its convexity forward. The anterior belly of the digastric muscle was broad and heavy; these fibers arose along the inferior border of the mandible. The anterior digastric formed a complete sheet of muscle extending from one premasseteric notch to that of the opposite side. There is no separation at the mid-line. These fibers passed posteriorly and slightly downward to attach to the convex side of the tendinous arch formed by the union of the two intermediate tendons. The fascia of the anterior belly of the digastric attached to that of the underlying mylohyoid muscle and joined the fascia of the submandibular gland. The intermediate tendon was connected to the hyoid bone by fascia that was not noticeably thickened.

Mylohyoid muscle.-The mylohyoid muscle lay deep to both the platysma and the anterior digastric muscles. It was a thin, delicate muscle, though its posterior border was definitely thickened. The mylohyoid muscle arose on the line extending from behind the last molar tooth to the inferior margin of the genioglossal fossa. From this origin the fibers passed downward and medially to insert into a raphe at the midline (Fig. 5). Only the posterior fibers of the mylohyoid attached to the hyoid bone. The 
raphe extended from the inferior margin of the genioglossal fossa to near the hyoid bone, where it spread out into a small triangular aponeurosis. Into this aponeurosis attached some of the middle fibers of the mylohyoid muscle.

The muscles described here are generally similar to those in man. A few points of differences, however, deserve special mention.

In the Macaque the temporalis muscle had a long tendon of insertion to the lower
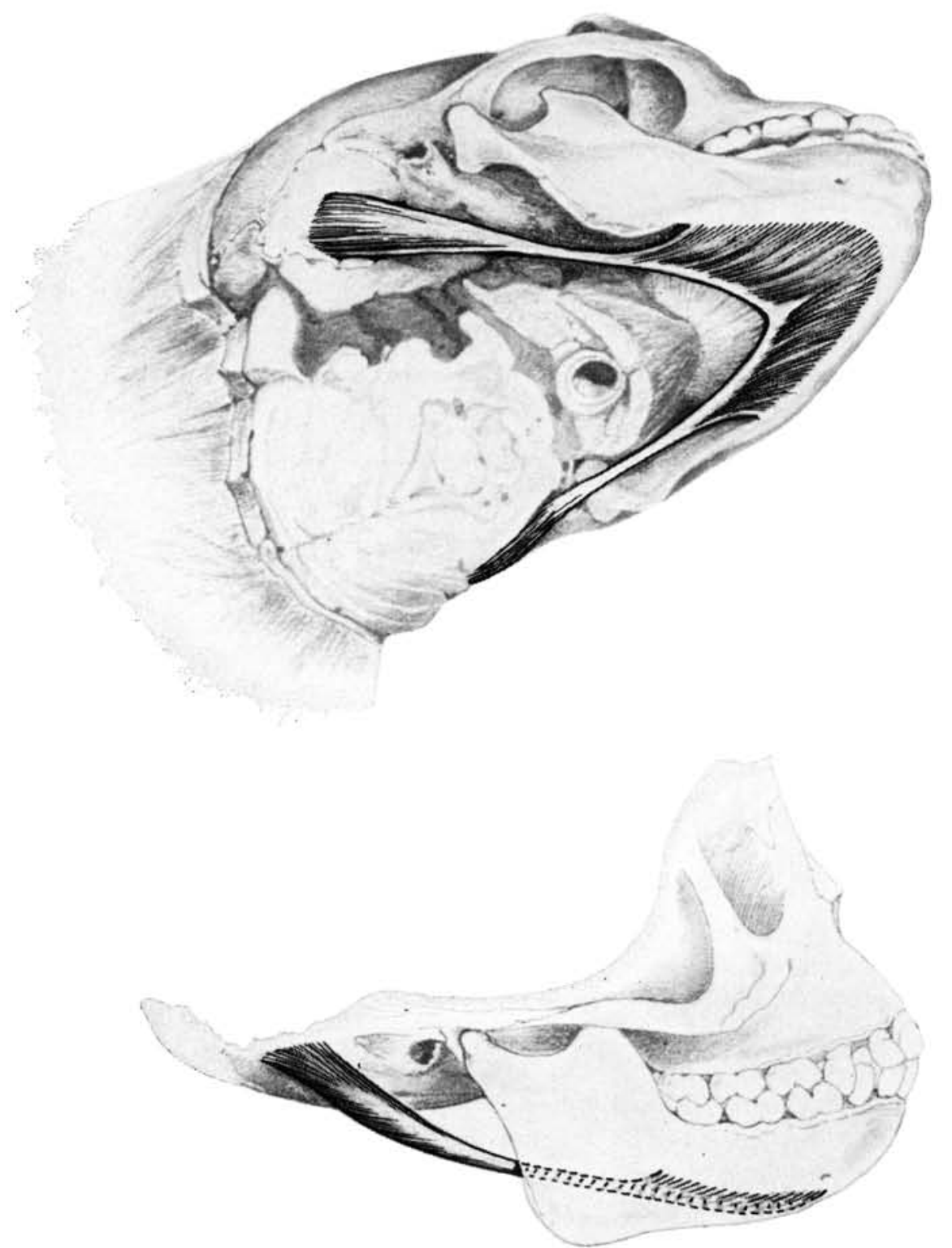

FIG. 4.-The digastric muscle. The continuous attachment of the anterior belly to the mandible from one side to another forms a "digastric sling." 
end of the oblique line, whereas this does not occur in man. The temporalis muscle attached not only to the ramus and coronoid process but also to most of the bone of the edge of the entire mandibular notch. The deep head of the masseter muscle was unusual, in that it had fibers of origin from the lateral and anterior surfaces of the temporomandibular joint capsule. Probably the most notable feature of these muscles was the continuity of one intermediate digastric tendon with the otherthe digastric sling-and the attachment of the anterior belly, without interruption, from one premasseteric notch to the other. The only characteristic feature of the mylohyoid muscle was the small triangular expansion of its mid-line raphe, forming an aponeurosis in front of the hyoid bone.

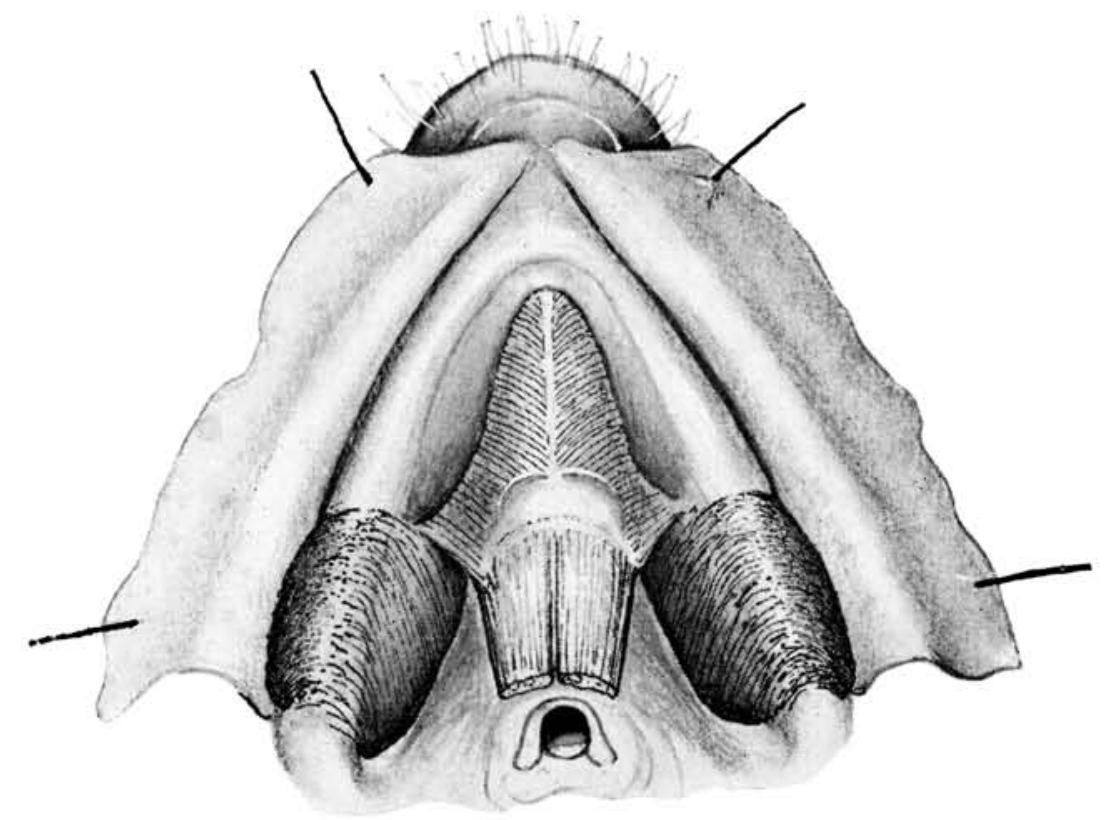

Fig. 5.-The mylohyoid muscle. The mid-line mylohyoid raphe expands posteriorly into an aponeurosis which attaches to the body of the hyoid bone. The posterior mylohyoid fibers attach directly into the hyoid bone, behind the aponeurosis.

The MANDIBULAR DIVISION OF TRIgEMINAL NERVE.-The mandibular nerve, the third division of the trigeminal nerve, arose from the trigeminal ganglion in the middle cranial fossa and passed downward a short distance to the foramen ovale. As the mandibular nerve passed through the foramen ovale, it divided into a smaller anterior division and a larger posterior division.

Anterior division of the mandibular nerve.-After arising from the mandibular nerve within the foramen ovale, the anterior division traveled forward and laterally, penetrating the lateral pterygoid plate via the pterygo-alar foramen, and entered the infra-temporal fossa, where it immediately divided into its terminal branches (Fig. 6).

A larger branch, the common root of the deep temporal and masseteric nerves, traveled directly laterally from the pterygo-alar foramen, crossing the upper surface of the lateral pterygoid muscle at a right angle, and divided just before reaching the 
medial surface of the ramus. One branch, a nerve to the temporalis muscle, entered the deep surface of the muscle at the level of the coronoid process (Fig. 7). The other branch, the masseteric nerve, passed behind the temporalis muscle through the posterior part of the mandibular notch and entered the masseter muscle on its deep surface.

The buccinator nerve, the largest branch of the anterior division of mandibular nerve, traveled forward and downward from the pterygo-alar foramen. It first passed medial to the superior head of the lateral pterygoid muscle and then curved laterally

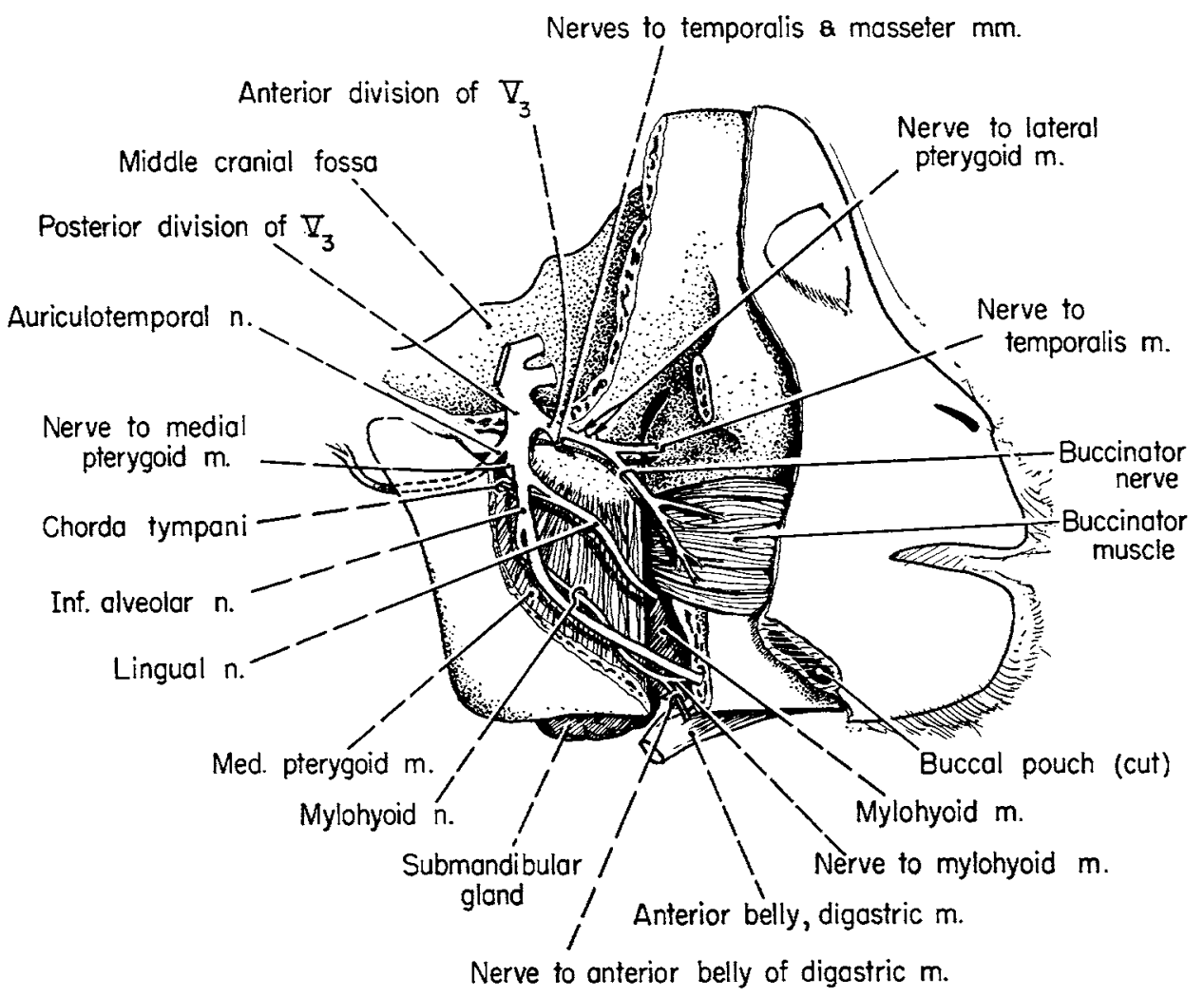

FIG. 6.-The mandibular division of the trigeminal nerve and its branches

between the upper and lower heads of this muscle (Fig. 7). In this passage, several small nerve filaments for the supply of both heads of the lateral pterygoid muscle arose from the buccinator nerve. In addition, a small fiber curved backward between the two heads of the lateral pterygoid muscle to reach the temporamandibular joint. The buccinator nerve then curved forward and downward, slightly below the base of the coronoid process, toward the center of the pterygomandibular raphe. The buccinator nerve passed through the temporalis muscle at about the mid-ramus level, and here it gave off a small anterior deep temporal branch to the temporalis muscle.

A small twig to the capsule of the temporomandibular joint passed posteriorly and laterally between the inferior surface of the temporal squama and the upper fibers 
of the lateral pterygoid muscle. This nerve reached the temporomandibular joint at about the center of the anterior surface of the joint capsule.

The buccinator nerve divided into two major branches just before reaching the pterygomandibular raphe. These branches immediately redivided and ramified through the cheek area.

Posterior division of the mandibular nerve.-The posterior division was the larger of the two major divisions of the mandibular nerve (Fig. 6). It arose from the mandibular nerve within the foramen ovale and, leaving the foramen, passed along the posterior edge of the lateral pterygoid plate, beneath the lateral pterygoid muscle.

The auriculotemporal nerve took origin from the posterior division immediately

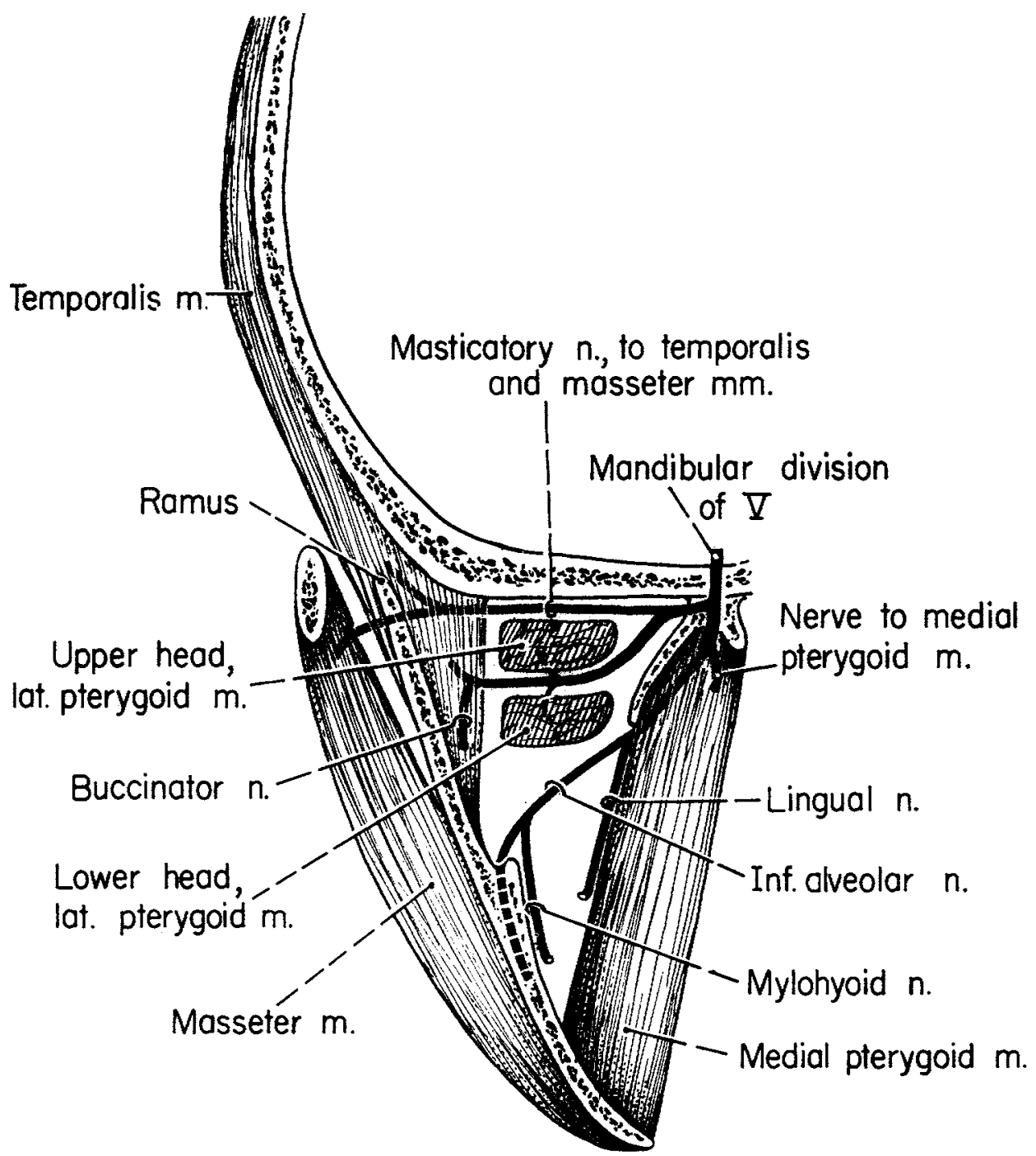

FIG. 7.-A diagrammatic frontal section through the ramus of the mandible, illustrating the relations of certain branches of the mandibular nerve. 
below the foramen ovale; it curved posteriorly, passing medial to the neck of the condyle. At the posterior border of the ramus, the nerve curved laterally through the parotid gland and passed upward in front of the ear. It divided into several branches in the auricular and temporal regions and in the parotid gland, supplying these areas (Figs. 6, 9).

The nerve to the medial pterygoid muscle arose from the posterior division, just below the origin of the auriculotemporal nerve, at the posterior border of the lateral pterygoid plate (Fig. 6). This nerve traveled a very short course and entered the medial pterygoid muscle high on its posteromedial surface, near the origin of the muscle from the pterygoid fossa.

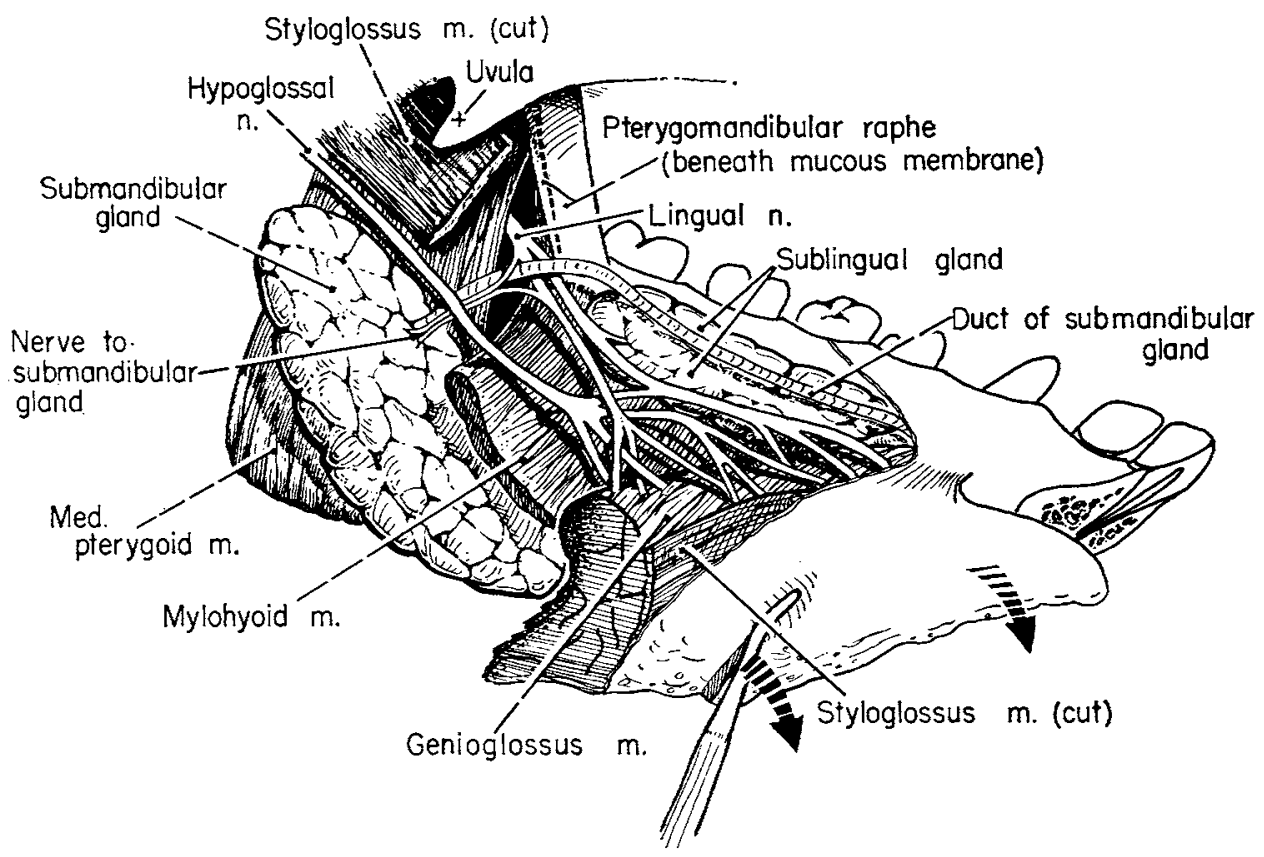

FIG. 8.-The lingual nerve and related structures in the paralingual space

The lingual nerve was one of the larger branches of the posterior division of the mandibular nerve. It arose a few millimeters below the nerve to the medial pterygoid muscle, at the level of the inferior border of the lateral pterygoid plate. As it began to curve forward on the lateral surface of the medial pterygoid muscle, the lingual nerve received the chorda tympani, which joined it at an acute angle immediately below the lateral pterygoid plate (Fig. 6). Continuing on the lateral surface of the medial pterygoid muscle, the lingual nerve slanted downward and forward to the anterior border of the muscle. Here the nerve turned medially about the posterior edge of the mylohyoid muscle. The lingual nerve then continued forward in the paralingual space between the tongue and the mylohoid muscle. It then passed beneath the duct of the submandibular gland. At first, lying above and lateral to the duct, the lingual nerve dipped beneath it to rise on the medial side of the duct and continue forward (Fig. 8). The nerve was tightly bound to the duct in the area of its loop 
beneath it, and here several very fine filaments left the lingual nerve to run with the duct or the submandibular gland, forming the nerve to the submandibular gland. No discrete submandibular ganglion was found. As the lingual nerve rose on the medial side of the submandibular duct, it continued forward in the paralingual space, running on the medial surface of the sublingual gland, to which it was tightly bound by connective tissue. A small branch was given off that entered the sublingual gland on its medial surface. Continuing forward, the lingual nerve passed between the sublingual gland and the genioglossus muscle, divided into several branches, and ramified into the tongue mass. Nearly all these twigs entered the body of the tongue between the genioglossus and styloglossus muscles. One of these twigs received a communication from the hypoglossal nerve. The lingual nerve was actually divided at the time of its loop beneath the submandibular duct. However, the branches were tightly bound together until they separated and ramified just before entering the tongue.
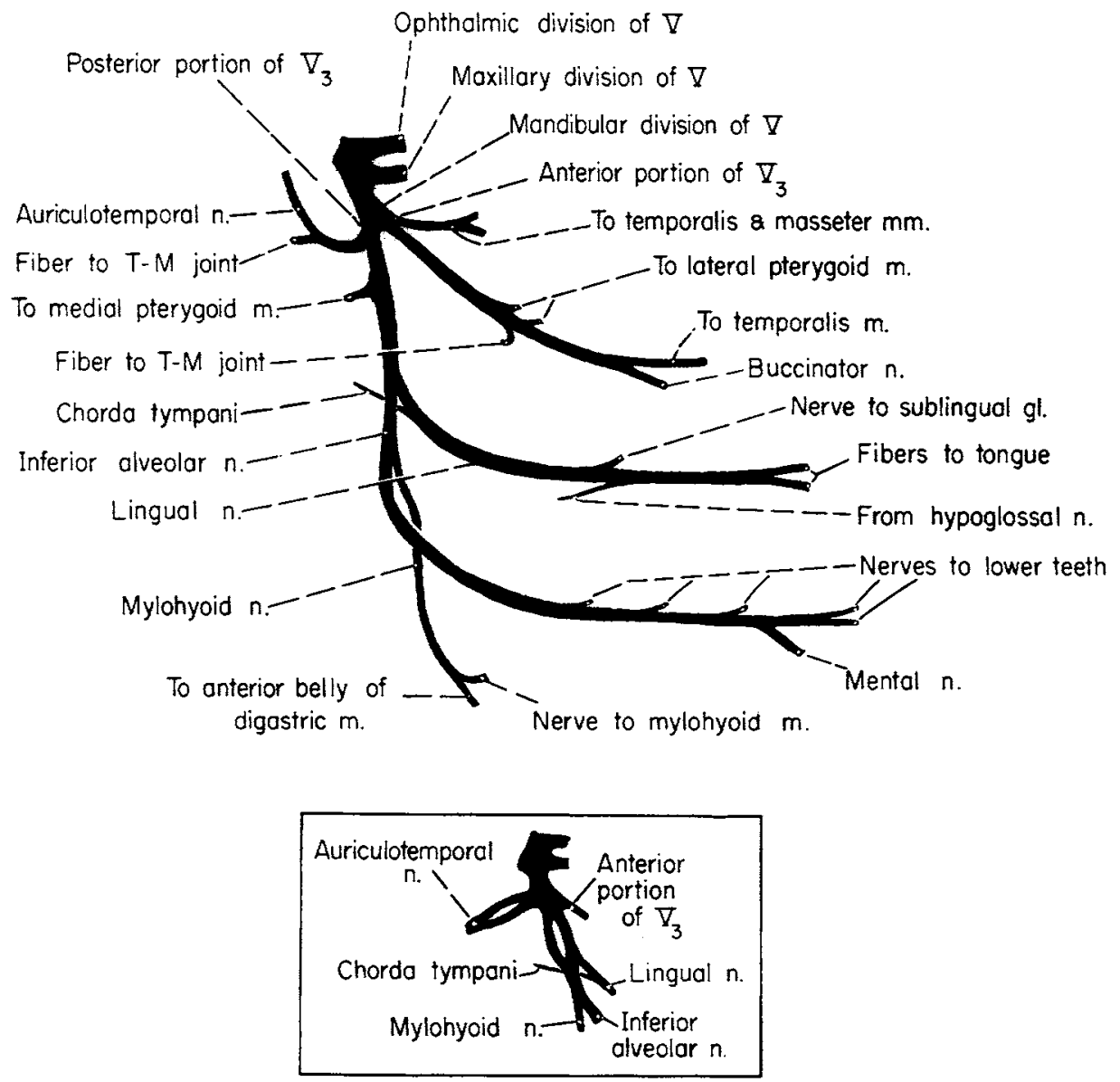

Fig. 9.-A diagrammatic sketch of the mandibular division and its branches. Inset: a splitting and re-formation of the auriculotemporal and inferior alveolar nerves ( 3 of 15 sides). 
The inferior alveolar nerve was a direct downward continuation of the posterior trunk; it passed between the ramus of the mandible and the medial pterygoid muscle to enter the mandibular foramen.* The inferior alveolar nerve ran within the bone of the mandible, in the mandibular canal, supplying the lower teeth. It gave off the mental nerve, which left the mandibular canal via the mental foramen to supply the skin, mucosa, and labial gingiva in the region of the anterior teeth.

The mylohyoid nerve arose from the inferior alveolar nerve just above the mandibular foramen and passed downward between the ramus and the medial pterygoid muscle. It lay in contact with the medial surface of the ramus, running in the very shallow mylohyoid sulcus. The mylohoid nerve curved forward, to emerge at the anterior border of the medial pterygoid muscle a few millimeters above the inferior border of the mandible. The nerve crossed the posterior border of the mylohyoid muscle and continued for a short distance between the lateral surface of the mylohyoid muscle and the anterior digastric muscle, before dividing into its terminal branches to the mylohyoid and anterior digastric muscles.

The mandibular nerve in the Macaque was seen to be very similar to that found in man. The consistency of the pterygo-alar foramen, through which the anterior division of the mandibular nerve passes, was striking. The mandibular division could not be visualized at the foramen ovale from the lateral view because of this bony bar extending posteriorly from the upper part of the lateral pterygoid plate. The nerve to the submandibular gland deserves special mention. These fibers were quite fine and were found along the duct of the gland. They were seen only with the dissection microscope and were verified by histologic section. No submandibular ganglion was identified.

\section{Summary}

The muscles of mastication and the accessory muscles of mastication in the Macaque were found to be generally similar to those found in man; the investigator using these forms for research purposes, however, must be aware of the differences that do exist. Only by knowing the detail in the anatomy of the laboratory animal can one interpret changes produced by experimental techniques.

We wish to express our thanks to Dr. Paul Ayres, of Parke, Davis and Company, Rochester, Michigan, and to the staff of the Department of Pathology, Parke, Davis and Company, Ann Arbor, Michigan, for their unstinting co-operation in supplying the many specimens used in this study.

\section{References}

1. Hartman, C. G., and Strauss, W. L. The Anatomy of the Rhesus Monkey. New York: Hafner Publishing Co., 1933.

* An interesting variation was observed in the origin of the auriculotemporal and inferior alveolar nerves on 3 of 15 sides. These nerves divided and then reformed to continue in their typical course. See insert, Fig. 9. 